\title{
Postural Sway Response to Local Vibratory Stimulation in Young, Middle-aged and Elderly People in Standing Position
}

\author{
Ayaka Yamada, Eishi Nakamura, Noritaka Sato, Yoshifumi Morita \\ Nagoya Institute of Technology \\ Gokiso-cho, Showa-ku, Nagoya, Aichi 466-8555, Japan \\ Tadashi Ito \\ International University of Health and Welfare \\ 2600-1 Kitakanemaru, Otawara, Tochigi 324-8501, Japan \\ Yoshihito Sakai \\ National Center for Geriatrics and Gerontology \\ 7-430, Morioka-cho, Obu City, Aichi 474-8511, Japan \\ Kazunori Yamazaki \\ Fujita Health University \\ 1-98 Dengakugakubo, Kutsukake-cho, Toyoake, Aichi 470-1192, Japan
}

E-mail:a.yamada.067@stn.nitech.ac.jp,e.nakamura.335@stn.nitech.ac.jp, sato.noritaka@nitech.ac.jp morita@nitech.ac.jp, tadashi.ito.0805@gmail.com, jsakai@ncgg.go.jp,ymzkk@fujita-hu.ac.jp

\begin{abstract}
We investigated the postural sway in response to local vibratory stimulation applied to young, middle-aged and elderly people in the standing position. For this purpose we developed a variable-frequency vibratory stimulation device and measured the postural sway using a gravicorder. As a result, when the vibratory stimulation was applied to the gastrocnemius muscles, the center of pressure moved backward in all subject groups. We found that elderly people with low back pain may perform balance control using their trunks more than their lower legs.
\end{abstract}

Keywords: Vibratory stimulation device, Proprioception, Postural sway, Low back pain, Fall prevention

\section{Introduction}

Falls accidents and low back pain in the elderly are two common problems in modern Japanese society. Up until 2009, over 1800 elderly people died from fall accidents per year. ${ }^{1}$ Low back pain is also a serious issue, but the causes are unidentifiable in $85 \%$ of patients. ${ }^{2}$ One possible factor is the problem of postural control involving voluntary and reflexive muscle responses, as suggested by one study in which it was reported that the elderly and people with low back pain have postural control problems involving deterioration of somatosensory receptors. ${ }^{3}$

To detect the motion of the body and adjust voluntary and reflexive muscle responses, the balance system uses not only sensory information from visual and vestibular senses but also somatosensory receptors ${ }^{4}$ that give superficial sensation and proprioceptive sensation. Moreover, proprioception in the leg and trunk muscles plays an important role in maintaining postural stability. ${ }^{5}$ Proprioceptive receptors have eigen response frequencies. Past studies have reported that when vibratory stimulations are applied, the postural sway in the standing position changes. ${ }^{6}$ Therefore, from the change of the postural sway during vibratory stimulations with various frequencies, we may be able to determine the cause of low back pain and falls and develop a treatment system for proprioception for recovery to solve these two problems.

On the other hand, other studies have reported that proprioception and vibration sensation in the lower legs 
decrease with aging. ${ }^{7}$ Moreover, postural instability has been observed in elderly people. ${ }^{8}$ That study and others, however, examined the postural sway of each age group in response to local vibratory stimulation applied using different vibratory stimulation devices under different experimental conditions.

Against such a background, the final goal of our research is to develop a treatment system for proprioception. To achieve this goal, the procedure involves investigating postural sway in response to local vibratory stimulation applied to human subjects in the standing position, to find the frequency of the vibratory stimulation and the body parts related to low back pain and falls, which will help to determine the causes of low back pain and falls.

In the work described in this paper, we developed a variable-frequency vibratory stimulation device and measured the postural sway response of young, middleaged, and elderly people to local vibratory stimulation when in the standing position. We focused on low back pain and investigated the frequencies of vibratory stimulation and the body parts related to low back pain.

\section{Variable-frequency vibratory simulation device}

We developed the variable-frequency vibratory stimulation device shown in Fig. 1. The device consists of a laptop computer, an audio amplifier, four vibrators, and a switch for selecting one of the vibrators. A sine wave signal with an arbitrary frequency generated on the laptop computer is input to the audio amplifier. By regulating the gain of the audio amplifier, the amplitudes of the vibrators can be changed. The range of displacement of the vibrators is $0-0.8 \mathrm{~mm}$, and the frequency range is $30-400 \mathrm{~Hz}$. Each vibrator is fixed to a holder. The four vibrators are attached to the left and right gastrocnemius muscles (GM) and the left and right lumbar multifidus (LM) with rubber belts. The lengths of the rubber belts are adjusted according to the perimeters of the body parts to which they are fixed to keep the pushing forces constant. As a gravicorder, we use a Wii Balance Board (Nintendo Co., Ltd.). The Wii Balance Board has been reported to be reliable enough for medical use. ${ }^{9}$

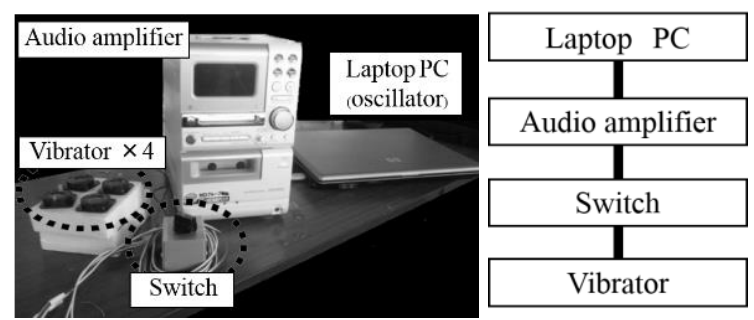

Fig.1. Photograph and block diagram of variable-frequency vibratory stimulation device.

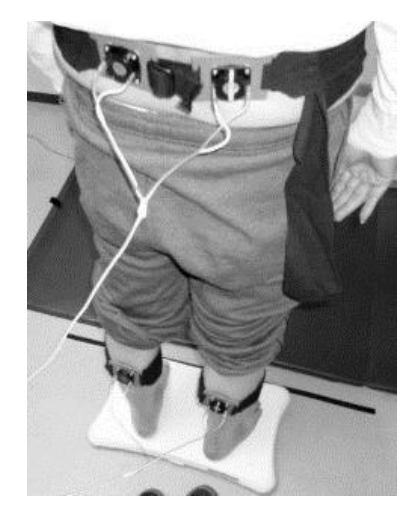

Fig.2. Subject wearing the device.

\section{Experiment}

\subsection{Purpose}

The purpose of the experiment was to investigate the postural sway response to local vibratory stimulation applied to young, middle-aged, and elderly people in the standing position by using the vibratory stimulation device that we developed, together with the Wii Balance Board.

\subsection{Subjects}

The subjects were 25 healthy young people ( 12 males and 13 females, aged $21.6 \pm 1.2$ ), 25 healthy middle-aged people (13 males and 12 females, aged $46.0 \pm 3.0$ ), 46 elderly people with lumbar spondylosis without low back pain ( 28 males and 18 females, age $=73.8 \pm 5.3$ ), and 28 elderly people with lumbar spondylosis with low back pain (10 males and 18 females, age $=75.5 \pm 5.1$ ). 
Table 1. Measurement procedure

(GM: Gastrocnemius muscles, LM: Lumbar multifidus)

\begin{tabular}{c|c|c}
\hline & Frequency & $\begin{array}{c}\text { Body part where vibratory } \\
\text { stimulation is applied }\end{array}$ \\
\hline Step 1 & $30 \mathrm{~Hz}$ & GM \\
Step 2 & $30 \mathrm{~Hz}$ & LM \\
Step 3 & $60 \mathrm{~Hz}$ & GM \\
Step 4 & $60 \mathrm{~Hz}$ & LM \\
Step 5 & $150 \mathrm{~Hz}$ & GM \\
Step 6 & $150 \mathrm{~Hz}$ & LM \\
Step 7 & $240 \mathrm{~Hz}$ & GM \\
Step 8 & $240 \mathrm{~Hz}$ & LM \\
\hline
\end{tabular}

\subsection{Method}

The center of pressure $(\mathrm{CoP})$ in postural sway while the subject stood still on the Wii Balance Board was measured as shown in Fig. 2. The subject stood barefoot on the Wii Balance Board with his/her feet together and his/her eyes open or closed. The subject was instructed to remain still and relaxed in the standing posture with his/her arms hanging loosely at his/her side.

One measurement for each subject consisted of eight steps for the different vibration frequencies and the different body parts where the vibratory stimulation was applied. The measurement procedure is shown in Table 1. An interval of $60 \mathrm{~s}$ was added after every step, during which each subject sat resting in a chair. Figure 3 shows the experimental procedure in one step. The time needed for one step was $75 \mathrm{~s}$. One step consisted of five sections, namely, "EO", "EC", "Pre", "Dur" and "Post". The time needed for one section was $15 \mathrm{~s}$. In the EO-section, the subject looked at a marker on the wall. In the EC-section and the Pre-section, the eyes were closed. Only in the Dur-section, the vibratory stimulation was applied to the subject with the eyes closed. In the Post-section, the subject's eyes were closed.

The vibratory stimulation was applied alternately to the two muscles (GM and LM). The amplitude of the vibration was set to $0.8 \mathrm{~mm}$. The frequencies were set to $30,60,150$, and $240 \mathrm{~Hz}$. It is well-known that the eigen response frequencies of Meissner's corpuscle, muscle spindle, and the corpuscle of Vater-Pacini are $30 \mathrm{~Hz}, 60$ $\mathrm{Hz}$, and $240 \mathrm{~Hz}$, respectively. ${ }^{10}$

\subsection{Experimental results}

We paid attention to the Pre-section and the Dur-section in order to analyze postural sway during local vibratory stimulation in the standing position. For this purpose we used two parameters related to the CoP, namely, the anterior movement of the $\operatorname{CoP}(\Delta M Y)$, and the relative proprioceptive weighting $(R P W)$ ratio. $\triangle M Y$ is defined as the difference between the mean values of the anterior

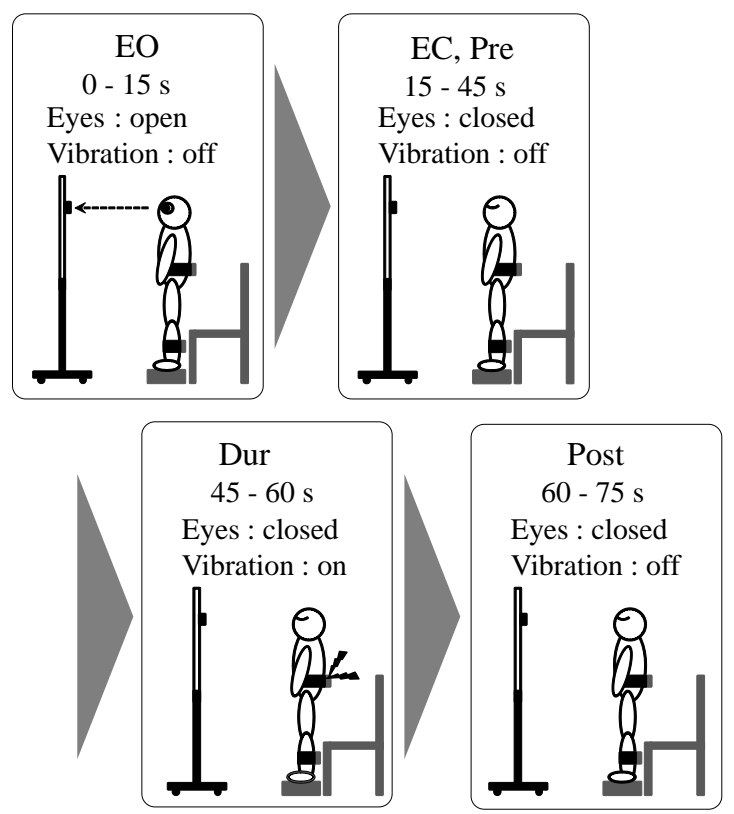

Fig.3. Experimental procedure.

displacement of the CoP in the Pre-section and the Dursection, which is given by

$$
\Delta M Y=M Y_{D u r}-M Y_{P r e},
$$

where

$$
\begin{aligned}
& M Y_{P r e}=\operatorname{Mean}_{P r e}\left\{Y_{C o P}(k)\right\} \\
& M Y_{D u r}=\operatorname{Mean}_{D u r}\left\{Y_{C o P}(k)\right\} \\
& Y_{C o P}(k)=y_{C o P}(k)-y_{C o P}(0)
\end{aligned}
$$

Here, $\operatorname{Mean}_{x x}\{\cdot\}$ denotes the mean of $\{\cdot\}$ during $X X$, $y_{C o P}(k)$ is the $y$-displacement of the $\mathrm{CoP}, k$ represents the sample index, and $y_{C o P}(0)$ is the value of $y_{C o P}(k)$ at the start of measurement. $\triangle M Y$ is calculated in each step. $\Delta M Y_{G M}$ and $\Delta M Y_{L M}$ denote the $\Delta M Y$ values for the gastrocnemius muscles and lumbar multifidus, respectively. The RPW ratio is defined by

$$
R P W=\frac{\left|\Delta M Y_{G M}\right|}{\left|\Delta M Y_{G M}\right|+\left|\Delta M Y_{L M}\right|} .
$$


As the $R P W$ ratio approaches $100 \%$, GM is used more than LM in motor control during quiet standing. This means that a lower leg-steered strategy is used in motor control. As the $R P W$ ratio approaches $0 \%$, this means that a trunk-steered strategy is used in motor control.

The experimental results are shown in Fig. 4, which shows the relationship between the frequency and $\triangle M Y$, and the relationship between the frequency and the $R P W$ ratio.

\subsection{Discussion}

Fig. 4(a), (d), (g) and (j) show that when the $60 \mathrm{~Hz}$ vibratory stimulation was applied to the GM, the CoP moved backward in all subject groups. This result is similar to the results obtained in previous studies 6,7 . Therefore, the proposed vibratory stimulation device and the experimental method using the developed device were confirmed to be effective in measuring the postural sway during local vibratory stimulation. Moreover, Fig. 4(a), (d), (g) and (j) show that when the vibratory stimulations with the frequencies of $30 \mathrm{~Hz}, 150 \mathrm{~Hz}$, and $240 \mathrm{~Hz}$ were applied to the GM, the CoP also moved backward in all subject groups.

On the other hand, Fig. 4(b), (e), (h) and (k) show that when the vibratory stimulations were applied to the LM, the CoP moved backward or frontward regardless of the change of frequency. This means that the biological response to vibration is uncertain.

Fig. 4(1) shows that as the frequency increased, the RPW ratio decreased. This response is a characteristic seen only in elderly people with low back pain. The same tendency was not seen in the other subject groups, as shown in Fig. 4(c), (f) and (i). This means that the RPW ratio does not depend on the frequency. Therefore, the results for the RPW ratios suggest that elderly people with low back pain performed balance control using their trunk more than their lower legs when vibratory stimulation with higher vibration frequency was applied.

\section{Conclusion}

In this paper, we investigated the postural sway in response to local vibratory stimulation applied to young, middle-aged, and elderly people in the standing position. For this purpose we developed a variable-frequency vibratory stimulation device and measured the postural sway according to our experimental method.
As a result, we found that our device and the experimental method were effective in measuring the postural sway during local vibratory stimulation. Moreover, from the result for the RPW ratio, we found that elderly people with low back pain used a trunksteered strategy in motor control.

Future work related to this study will be to analyze the relationship between the postural sway and falls, to determine the causes of low back pain and falls through additional experiments, and to develop a treatment system for proprioception.

\section{References}

1. VITAL STATISTICS, Statistics and Information Department, Minister's Secretariat, Ministry of Health, Labor and Welfare JAPAN, http://www.mhlw.go.jp/english/database/dbhw/index.html

2. A. Richard, R. James and L. Daniel, What can the history and physical examination tell us about low back pain? J. the Am. Med. Assoc. 268 (1992) 760-765.

3. T. Urushihata and T. Kinugasa, Aging effects on the structure underlying balance abilities tests, Tairyokukagaku 56(6) (2007) 711. (In Japanese)

4. R. Johansson and M. Magnusson, Human postural dynamics Crit. Rev. Biomed. Eng. 18 (1991) 413-437.

5. B. Bloem, J. Allum, M. Carpenter and F. Honegger, Is lower leg proprioception essential for triggering human automatic postural responses?, Experimental Brain Research 130 (2000) 375-391.

6. B. Simon, C. Paul and V. Sabine, Proprioceptive weighting changes in persons with low back pain and elderly persons during upright standing Neuroscience Letters. 366 (2004) 63-66.

7. B. Skinner, L. Barrack and D. Cook, Age-related decline in proprioception Arch. Phys. Med. Rehabil. 44 (1963) 645-650.

8. I. Pyykkö, P. Jäntti and H. Aalto, Postural control in elderly subjects, Age Aging. 19 (1990), 215-221.

9. A. Clark and L. Bryant, Validity and reliability of the Nintendo Wii Balance Board for assessment of standing balance Gait \& Posture. 31 (2010) 307-310.

10. T. Ito, Y. Sakai, A. Kubo, K. Yamazaki, Y. Ohno, E. Nakamura, N. Sato, Y. Morita. The relationship between physical function and postural sway during local vibratory stimulation of middle-aged people in the standing position J. Phys. Ther. Sci. 26(10) (2014) 1627-1630. 


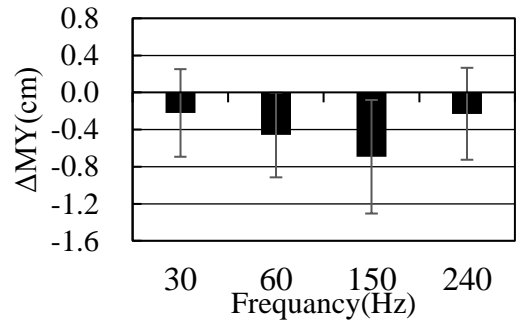

(a) $\triangle \mathrm{MY}$ with stimulation of GM in healthy young people.

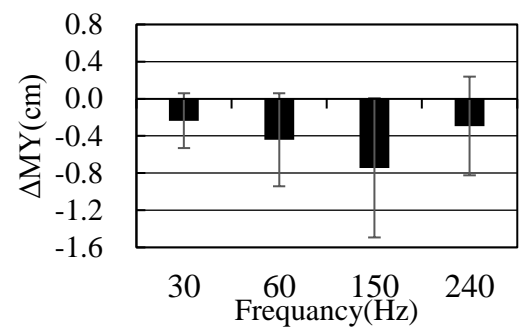

(d) $\triangle \mathrm{MY}$ with stimulation of $\mathrm{GM}$ in healthy middle-aged people.

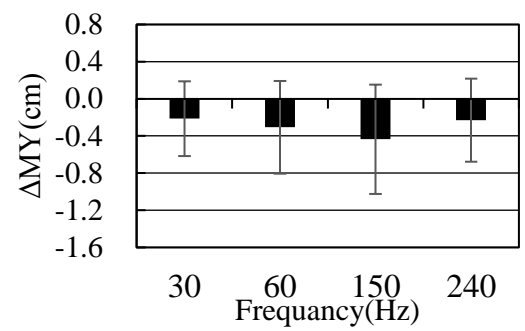

(g) $\triangle \mathrm{MY}$ with stimulation of $\mathrm{GM}$ in elderly people without low back pain.

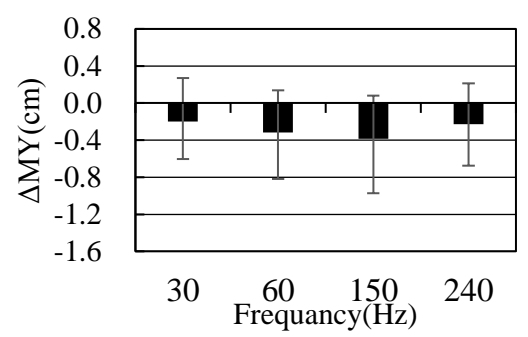

(j) $\triangle \mathrm{MY}$ with stimulation of $\mathrm{GM}$ in elderly people with low back pain.

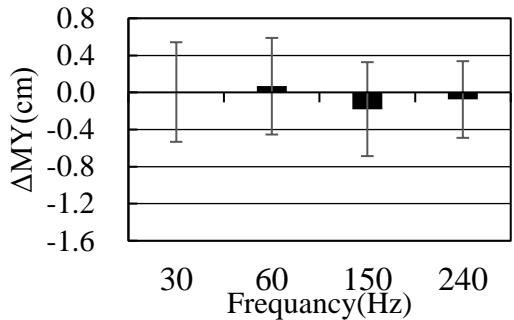

(b) $\triangle \mathrm{MY}$ with stimulation of LM in healthy young people.

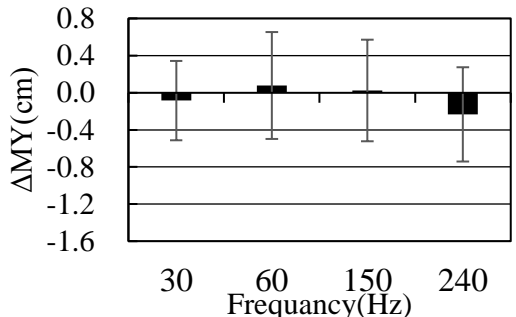

(e) $\triangle \mathrm{MY}$ with stimulation of LM in healthy middle-aged people.

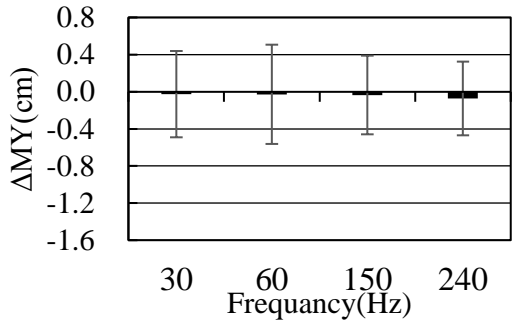

(h) $\triangle \mathrm{MY}$ with stimulation of LM in elderly people without low back pain.

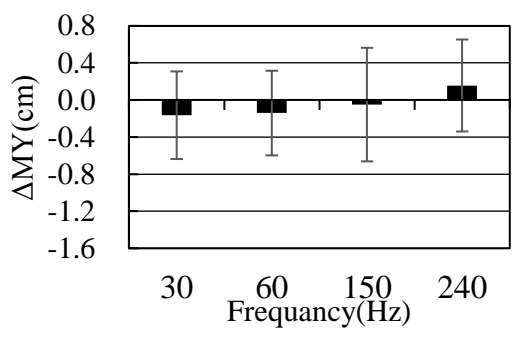

(k) $\triangle \mathrm{MY}$ with stimulation of $\mathrm{LM}$ in elderly people with low back pain.

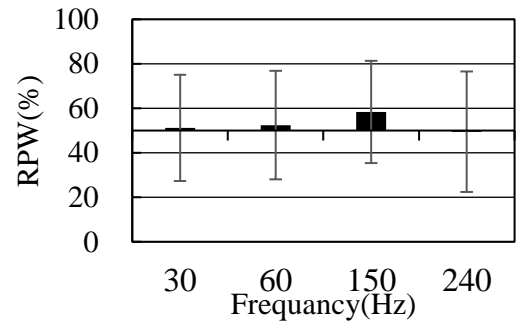

(c) RPW ratio in healthy young people.

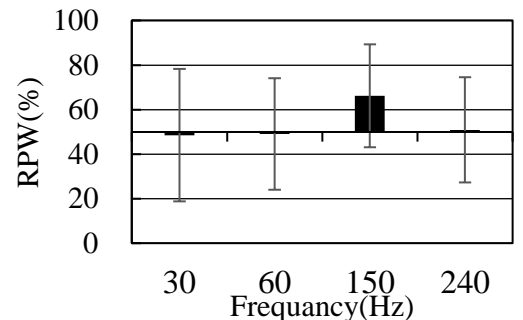

(f) RPW ratio in healthy middle-aged people.

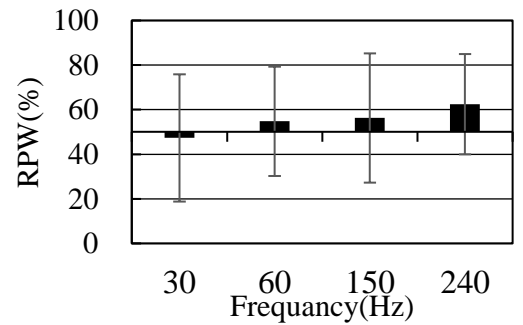

(i) RPW ratio in elderly people without low back pain.

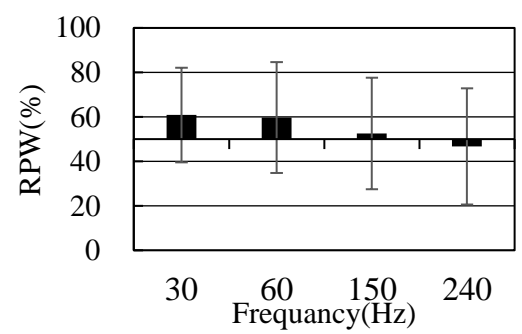

(1) RPW ratio in elderly people with low back pain.

Fig.4. Frequency dependence of $\triangle \mathrm{MY}$ and RPW ratio. 